\title{
GERMINAÇÃO DE SEMENTES E INOCULAÇÃO DE MUDAS DE MACACAÚBA (Platymiscium trinitatis Benth. - LEGUMINOSAE PAPILIONOIDEAE) COM RIZÓBIOS EM LATOSSOLO AMARELO
}

\author{
Luiz Augusto Gomes de SOUZA', Marlene Freitas da SILVA ${ }^{2}$, Adilson \\ Rodrigues DANTAS ${ }^{1}$
}

\begin{abstract}
RESUMO - Foram obtidas informações silviculturais sobre a macacaúba - Platymiscium trinitatis Benth (Leguminosae Papilionoideae), abordando aspectos da germinação das sementes e do efeito da inoculação com rizóbios na formação de mudas. A semeadura foi efetuada em areia, acompanhada por 45 dias, e as plântulas repicadas para sacos com latossolo amarelo coletado após a queima da vegetação, no horizonte $\mathrm{A}(0-20 \mathrm{~cm})$, distribuido em recipientes plásticos com capacidade para 2,0 $\mathrm{kg}$ de solo. As mudas foram submetidas a tratamentos de adubação com $\mathrm{N}$ mineral $(50 \mathrm{~kg} / \mathrm{ha})$ ou a inoculação com estirpes de rizóbios da coleção do INPA/CPCA. O desenvolvimento das mudas foi acompanhado com avaliação mensal do comprimento do caule e diâmetro do colo das plantas. Aos 126 dias estas foram colhidas e avaliadas. As sementes apresentaram elevada viabilidade com $86 \%$ de germinação, iniciada aos 4 dias e distribuindo-se por 37 dias. O Índice de Velocidade de Emergência foi de $21,9(n=200)$. A repicagem das plantas para sacos pode ser feita em 40 dias. Os rizóbios utilizados como inoculante formaram colônias brancas, com até $4 \mathrm{~mm}$ de diâmetro após a incubação, com características morfológicas e culturais bastante variadas. No viveiro as mudas apresentaram crescimento lento e não apresentaram resposta à inoculação, o que foi relacionado aos níveis elevados de matéria orgânica presentes, o que, entretanto, não favoreceu o seu desenvolvimento. $\mathrm{O}$ incremento mensal de comprimento do caule e diâmetro do colo das plantas foi de $2,49 \mathrm{~cm}$ e $0,45 \mathrm{~mm}$, respectivamente.
\end{abstract}

Palavras-Chave - Platymiscium trinitatis, sementes, germinação, inoculação, rizóbios, macacaúba, Leguminosae.

Seed Germination and Plant Inoculation of Macacaúba (Platymiscium trinitatis Benth. Leguminosae Papilionoideae) with Rhizobia in a Yellow Latossol.

ABSTRACT - This paper presents silvicultural information about "macacaúba" (Platymiscium trinitatis Benth., Leguminosae, Papilionoideae), including information on seed germination and the effect of rhizobia inoculation in the nursery. Seeds were sown in sand, germination characteristics observed for 45 days, and the seedlings were transplanted to recipients with an oxisol (latossolo amarelo), collected in the "A" horizon $(0-20 \mathrm{~cm})$, after the slash and burn of the vegetation, in plastic bags with $2.0 \mathrm{~kg}$ capacity. The plants received $\mathrm{N}$ mineral fertilization $(50 \mathrm{~kg} / \mathrm{ha})$ or were inoculated with rhizobia strains from the INPA/CPCA collection. Plant development was evaluated monthly by measuring height and diameter at soil level. After 126 days in the nursery, the plants were collected and evaluated. The seed had high viability, with $86 \%$ germination, beginning four days after sowing and distributed over 37 days. The emergence velocity index (EVI) was $21,9(n=200)$. Transplanting to nursery bags can be done at 40 days. The rhizobia used for inoculation developed white colonies, $4 \mathrm{~mm}$ in diameter after incubation, with variable morphological and cultural characteristics. In the nursery, the plants presented slow growth and no rhizobia response, possibly due to the organic matter levels in the substrate being unsuitable for good development of the plants. The monthly height and diameter increments of the plants were $2,49 \mathrm{~cm}$ and $0,45 \mathrm{~mm}$, respectively.

Key-Words - Platymiscium trinitatis, seeds, germination, inoculation, Rhizobia, macacaúba, Leguminosae.

${ }^{1}$ Instituto Nacional de Pesquisas da Amazônia, Coordenação de Pesquisas em Ciências Agronômicas. Caixa Postal 478,69.011-970 Manaus, AM, Brasil e-mail: souzalag@inpa.gov.br ${ }^{2}$ Universidade Tecnológica da Amazônia, Departamento de Engenharia Florestal. Manaus, AM. 


\section{INTRODUÇÃO}

A macacaúba ou macacaúba da terra firme (Platymiscium trinitatis Benth., Leguminosae, Papilionoideae) é uma espécie madeireira distribuída em toda a Amazônia, de elevada importância comercial nos mercados nacionais e internacionais. Por seu valor econômico, as informações silviculturais sobre esta espécie são de grande interesse, já que ela pode tornar-se uma espécie à ser considerada para plantios florestais na região. Várias espécies do mesmo gênero, tais como $P$. ulei Harms no Brasil, $P$. pinnatum (Jacq.) Dugand no Panamá e Venezuela, e $P$. dimorphandrum Donn Smith na Guatemala são importantes árvores madeireiras (Allen \& Allen, 1981). Entretanto, pouco se sabe sobre a silvicultura das espécies do gênero, o que ainda demanda estudos experimentais que gerem informações básicas de autoecologia.

A nodulação em macacaúba foi reportada recentemente por Moreira et al. (1992), que verificaram a presença de nódulos estabelecidos no sistema radicular de plantas desta espécie. Atualmente, considera-se que a fixação biológica de nitrogênio, pela simbiose entre rizóbios e leguminosas, seja o processo mais eficiente de adição de $\mathrm{N}$ ao sistema solo/planta/animal (Franco \& Silva, 1985). Tal fato tem permitido que a tecnologia desenvolvida para o uso de rizobactérias como inoculante de leguminosas esteja se expandindo para espécies de interesse florestal, abrindo novas possibilidades de aproveitamento desta simbiose, especialmente na combinação de espécies em

sistemas agrosilviculturais auto-sustentáveis em N (Franco \& Faria, 1997).

O principal interesse na prática da inoculação em leguminosas arbóreas na fase de formação das mudas está em efetuar o plantio da espécie de interesse com os nódulos estabelecidos, se possível pela inoculação de rizóbios selecionados. Tem sido verificado em leguminosas florestais noduliferas que tal prática permite que as plantas retomem rapidamente o seu desenvolvimento após o plantio, favorecidas pela autosuficiência em nitrogênio (Dobereiner, 1967; Ribeiro Júnior, 1985).

Este estudo foi efetuado com a finalidade de se obter maiores informações sobre a germinação das sementes e propriedades silviculturais da macacaúba, bem como para avaliar o efeito da inoculação com rizóbios nas mudas, durante a fase viveiro, em substrato argiloso.

\section{MATERIAL E MÉTODOS}

Os estudos foram desenvolvidos no Laboratório de Microbiologia do Solo e no viveiro do Instituto Nacional de Pesquisas da Amazônia, INPA, Manaus, AM, Brasil.

As sementes da macacaúba foram coletadas no mês de abril de uma matriz adulta, cultivada, no Campus do INPA, que atinge $11 \mathrm{~m}$ de altura e tem circunferência a altura do peito de 
1,61 m. Nesta época do ano, os frutos encontravam-se em dispersão por anemocoria e foram coletados em um raio de 30-40 m de distância da matriz. Após a coleta, 300 frutos foram separados para o teste de pureza (utilizando-se três repetições de 100 sementes) e determinação do peso de 1.000 sementes e do número de sementes por quilo. $\mathrm{O}$ teor de umidade das sementes foi determinado após secagem em estufa à $105^{\circ} \mathrm{C}$, por 24 horas. Foram também efetuadas determinações biométricas em frutos e sementes, tomando-se medidas de comprimento, largura e espessura, com 10 repetições.

Após o beneficiamento manual dos frutos, 200 sementes foram mantidas imersas em água, em temperatura ambiente $\left( \pm 26^{\circ} \mathrm{C}\right)$, por 24 horas, seguindo-se a semeadura em substrato de areia lavada, utilizando-se um delineamento experimental de quatro repetições de 50 sementes. Como cobertura de semeio utilizou-se uma camada de $0,5 \mathrm{~cm}$ de casca de arroz.

Como critério de germinação, adotou-se a emergência do caulículo. Acompanhou-se a emergência das plântulas diariamente, mantendo-se o nivel de umidade do substrato com irrigações periódicas. Ao final do período, foram calculadas a porcentagem de germinação e Índice de Velocidade de Emergência (IVE), segundo Popinigis (1977).

As plantas remanescentes deste teste foram repicadas aos 41 dias após a semeadura, utilizando-se a técnica das raizes nuas. O solo utilizado foi classificado como latossolo amarelo, textura muito argilosa, coletado no Distrito Agropecuário da SUFRAMA ( $2^{\circ} 30^{\prime} \mathrm{S}$ e $\left.60^{\circ} 00^{\prime} \mathrm{W}\right)$, localizado no $\mathrm{km}$ 62 da BR 174, um mês após a queima da vegetação com fins de preparo agrícola da terra. Somente solo do horizonte $\mathrm{A}(0-20 \mathrm{~cm})$ foi utilizado. Após a coleta, o solo foi destorroado e peneirado em peneira metálica de $2 \mathrm{~cm}$ de malha. Como recipiente para o desenvolvimento das mudas foram utilizados sacos de polietileno preto com capacidade para $2,0 \mathrm{~kg}$ de solo. Uma amostra do substrato foi separada para determinações químicas, que foram efetuadas no Laboratório de Solos da EMBRAPA/CPAA, em Manaus, utilizado a metodologia da EMBRAPA (1979). O solo utilizado apresentava acidez média ( $\mathrm{pH} 5,5)$ e teores de cálcio, magnésio e alumínio de $3,05,1,10$ e $0,30 \mathrm{cmol}_{\mathrm{c}} / \mathrm{dm}^{3}$, respectivamente. O conteúdo de fósforo e potássio encontrado foi de 18 e $108 \mathrm{mg} / \mathrm{dm}^{3}$, e os teores de carbono e de matéria orgânica foram $3,63 \%$ e $6,25 \%$, respectivamente.

Cinco estirpes de rizóbios que fazem parte da coleção do INPA (Souza, 1996) foram utilizadas neste estudo: $512,524,525,541$ e 590 . Estas estirpes foram isoladas de espécies dos gêneros Platymiscium e Pithecellobium. Estas estirpes tiveram sua autenticidade confirmada em testes conduzidos sob condições monoaxênicas utilizando siratro (Macroptilium atropurpureum (DC) Urban) como planta teste. Algumas características culturais e morfológicas destas estirpes (Sommasegaran \& Hoben, 1985), tais como cor e 
tamanho das colônias, consistência, velocidade de crescimento, tendência a coalescência e a produção de exopolissacarídeos, foram utilizados para caracterizar as bactérias aqui avaliadas. De modo geral, as estirpes utilizadas como inoculante apresentaram colônias brancas, atingindo 2-4 $\mathrm{mm}$ de diâmetro na superfície do meio de cultura após a incubação (Tab. 1) e todas foram infectivas quando cultivadas em condições axênicas, condição indispensável para classificação destas como do grupo dos rizóbios (Pelczar et al., 1980).

O desenho experimental foi o inteiramente casualizado, com sete tratamentos e 10 repetições. As médias foram comparadas pelo modelo de Tukey. Os tratamentos foram: testemunha, com plantas não inoculadas e não adubadas com nitrogênio sintético; plantas não inoculadas e adubadas com nitrogênio sintético $(0,16 \mathrm{~g}$ de $\mathrm{N}$-uréia por saco, correspondente a $50 \mathrm{~kg} /$ ha de $\mathrm{N}$-uréia); tratamentos de inoculação individual com as estirpes INPA 512, INPA 524, INPA 525, INPA 541 e INPA 590.

O acompanhamento do crescimento das plantas foi feito com medições do comprimento do caule e diâmetro do colo das mudas, efetuado aos $31,64,101$ e 126 dias após a repicagem. Aos 126 dias procedeuse a colheita das mudas, separandoas em parte subterrânea e aérea. Foram determinados o peso de biomassa fresca e seca da parte aérea das mudas, o peso seco das raizes e nódulos, a matéria seca total das plantas, o número de nódulos, a cor das folhas e a relação raiz/parte

Tabela 1. Características culturais e do hospedeiro de origem das estirpes de rizóbios utilizadas como inoculante da macacaúba (Platymiscium trinitatis Benth.).

\begin{tabular}{|c|c|c|c|}
\hline$N^{2}$ da estirpe & Hospedeiro de origem & Características culturais & Grupo de rizóbio \\
\hline INPA 512 & Platymiscium trinitatis & $\begin{array}{l}\text { Colônias branco-gelo, de crescimento lento } \\
\text { * } 1 \text {, butirosas, comaté } 2 \mathrm{mmde} \text { diâmetro, } \\
\text { coales centes, sem produçäo de } \\
\text { exopolissacarídeos. }\end{array}$ & Bradyrhizobium \\
\hline INPA 524 & Platymiscium ulei & $\begin{array}{l}\text { Colônias brancas, de crescimento } \\
\text { intermediário, com } 2 \mathrm{~mm} \text { de diâmetro, } \\
\text { viscosas, coalescentesecomabundante } \\
\text { produçăo de exopolissacarideos. }\end{array}$ & Bradyrhizobium \\
\hline INPA 525 & Platymiscium ulei & $\begin{array}{l}\text { Colônias branco-leitosas, aquosas, de } \\
\text { crescimento intermediário, com } 2 \text { mmde } \\
\text { diâmetro, coalescentes e com pouca } \\
\text { produçåo de exopolissacarídeos. }\end{array}$ & Bradyrhizobium \\
\hline INPA 541 & Pithecellobium saman & $\begin{array}{l}\text { Colônias brancas, translúcidas, de } \\
\text { crescimento intermediário, com } 3 \mathrm{~mm} \text { de } \\
\text { diâmetro, aquosas, coalescentes e com } \\
\text { pouca produçăo de exopolissacarídeos. }\end{array}$ & Bradyrhizobium \\
\hline INPA 590 & Pithecellobium saman & $\begin{array}{l}\text { Colôniasbranco-leitosasdecrescimento } \\
\text { rápido, aquosas, com } 4 \mathrm{~mm} \text { de diâmetro, } \\
\text { coalescentes e com muita produçâo de } \\
\text { exopolissacarideos. }\end{array}$ & Rhizobium \\
\hline
\end{tabular}

"- Crescimento rápido: 3-5 dias, intermediário: 5-10 dias, e lento: superior a 10 dias. 
aérea das mudas. A desidratação do material foi feita em estufa de incubação à $65^{\circ} \mathrm{C}$ por 72 horas. $\mathrm{O}$ cálculo mensal de incremento do crescimento das plantas foi feito segundo Benincasa (1988).

\section{RESULTADOS E DISCUSSÃO}

Os frutos de "macacaúba" apresentaram as seguintes propriedades silviculturais: porcentagem de pureza $58,20 \pm 0,70 \%$; número de sementes por quilo $-2.029 \pm 124$ sementes; peso de 1.000 sementes $-494 \pm 30$ g. Pasztor (1963), em frutos de sacambu (Platymiscium floribundum Vog.), da mata Atlântica do Brasil, encontrou taxa de pureza de $75-80 \%$, enquanto o número de sementes por quilograma variou entre 1.200 a 1.500 .

Os frutos de macacaúba apresentavam comprimento de 7,9 \pm $1,1 \mathrm{~cm}$, largura de $3,6 \pm 0,4 \mathrm{~cm}$ e espessura de $0,20 \pm 0,06 \mathrm{~cm}$, pesando individualmente $0,614 \pm$ $0,157 \mathrm{~g}$. As sementes apresentavam $9,69 \%$ de umidade e dimensões de 2,2 $\pm 0,2 \mathrm{~cm}$ de comprimento, $1,3 \pm 0,2 \mathrm{~cm}$ de largura e $0,16 \pm 0,04 \mathrm{~cm}$ de espessura, com peso médio individual de $0,30 \pm 0,13 \mathrm{~g}$. Corrêa (1926) mediu os frutos de $P$. duckei var. nigrum, encontrando de $6-7 \mathrm{~cm}$ de comprimento e $3,5 \mathrm{~cm}$ de largura. A mesma espécie, segundo Moreira \& Moreira (1996), tem sementes com $2,1 \mathrm{~cm}$ de comprimento, o que concorda com os resultados aqui apresentados.

A germinação das sementes teve inicio aos 4 dias após a semeadura e distribuiu-se por um período germinativo de 37 dias, com indice final de $86,0 \%$. A emergência das plântulas é do tipo epígea, fanerocotilar. O IVE foi de 21,9 (n = 200). Moreira \& Moreira (1996) também encontraram uma elevada taxa germinativa para macacaúba, de $92,0 \%$, iniciando-se aos 3 dias após a semeadura e estendendo-se até os 28 dias. Contrariamente, SUDAM/ CMT (1979) registrou que as sementes de Platymiscium trinitatis apresentaram baixo poder germinativo.

Neste trabalho, a germinação das sementes ocorreu principalmente nos primeiros 12 dias após a semeadura, ocorrendo posteriormente mais algumas emergências de plântulas, até os 41 dias (Fig. 1). Pôde-se constatar que aos 12 dias após a semeadura, $37,5 \%$ das sementes germinaram, evidenciando um pico da germinação das sementes. É possivel obter boa e eficiente germinação natural de sementes de macacaúba, o que favorece o seu aproveitamento silvicultural.

O crescimento das mudas não foi favorecido pela inoculação com rizóbios e houve baixa resposta das mudas à adubação com N-uréia (Tab. 2), o que foi atribuído ao elevado nivel de fertilidade do substrato. Aos 126 dias de enviveiramento, as plantas haviam desenvolvido em média 18,1 $\mathrm{cm}$ de comprimento do caule e 3,2 $\mathrm{mm}$ de diâmetro do colo, e encontravam-se com qualidade adequada para o plantio definitivo. $\mathrm{O}$ incremento em comprimento do caule das plantas correspondeu a $2,49 \mathrm{~cm}$ ao 


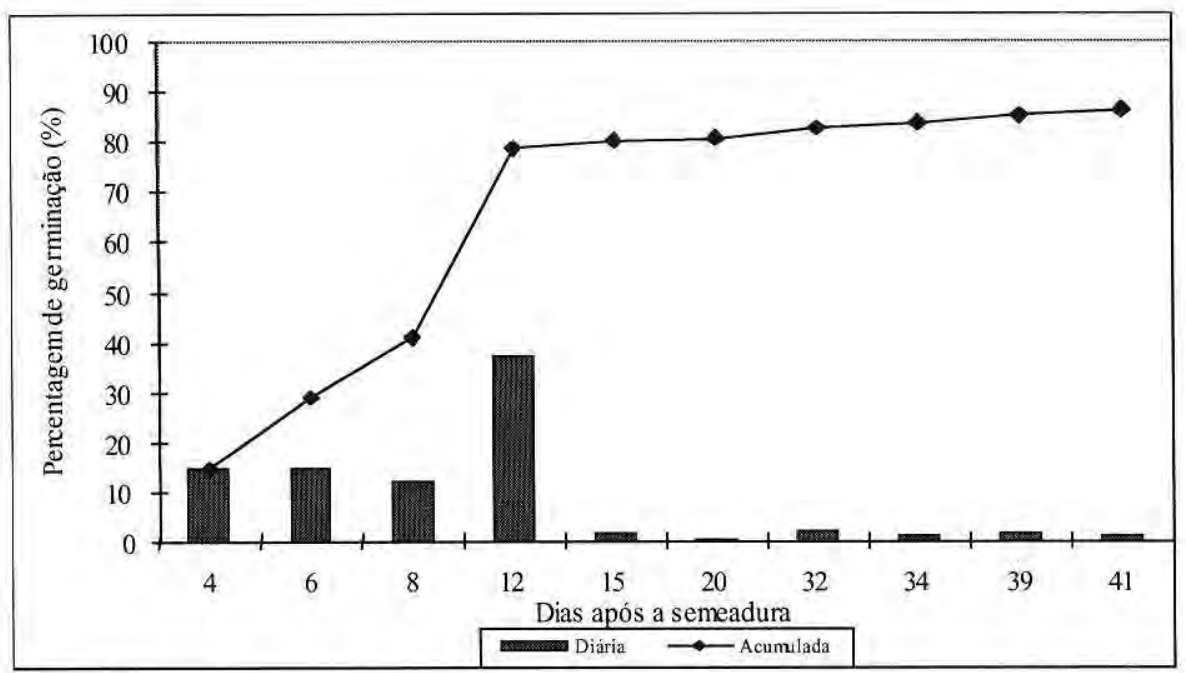

Figura 1. Curva da germinação natural das sementes de macacaúba (Platymiscium trinitatis).

mềs. Nas condições do ensaio o periodo de enviveiramento desta espécie pode ser planejado para cerca de 4-5 meses.

O crescimento em diâmetro do colo das mudas de macacaúba foi pouco afetado pelos tratamentos aplicados neste experimento (Tab. 2). Constatou-se que, aos 31 dias após a inoculação, variações no diâmetro do colo das plantas $(\mathrm{P}<0,05)$, de modo que as mudas inoculadas com a estirpe INPA 512 apresentavam maior diâmetro que aquelas tratadas com a estirpe INPA 525 ou com N mineral. Estas diferenças entretanto não se mantiveram ao longo do ensaio e as plantas passaram à ter uniformidade nas avaliações realizadas aos 64 e 101 dias. Por ocasião da colheita as mudas que receberam $\mathrm{N}$-mineral encontravam-se com diâmetro do colo maior que aquelas inoculadas com a estirpe INPA 525. A média diamétrica das plantas que receberam nitrogênio mineral não diferiu do tratamento controle. A taxa de incremento diamétrico foi estimada em $0,45 \mathrm{~mm}$ ao mês, resultado que possibilita classificar esta planta como de crescimento lento, na fase de formação das mudas (Atik, 1992).

A biomassa das plantas de macacaúba inoculadas com rizóbios na fase viveiro também não foi afetado pelos tratamentos empregados (Tab. 3). Aos 126 dias, as mudas apresentaram entre 1,42 e $2,22 \mathrm{~g}$ de matéria seca total. Ao final do experimento verificou-se que a taxa de sobrevivência das mudas foi de $95,7 \%$.

As mudas de macacaúba inoculadas com rizóbios não responderam à inoculação, apresentando nodulação irregular ao final do experimento, quando somente $16,3 \%$ das plantas encontravam-se noduladas (Tab. 4). Inoculadas com a 
estirpe homóloga INPA 512, as plantas não nodularam (Tab. 4). A estirpe INPA 590, isolada de Pithecellobium saman, também não foi infectiva. Por outro lado, a presença de plantas noduladas em tratamentos não inoculados indicou a presença de rizóbio nativo neste solo, embora com baixo potencial de infectividade.

Os nódulos observados em macacaúba apresentam forma esférica, com coloração variando entre vermelho (os mais jovens) e marrons (os mais antigos). As raízes da macacaúba também apresentam cor variável entre o creme, marrom e vermelho. Nas plantas onde constatou-se a formação de nódulos, estes variaram em média de 2 a 15 por planta, com maior freqüência numérica de mudas noduladas no

Tabela 2. Efeito da inoculação com rizóbios e da adubação nitrogenada no crescimento de mudas de macacaúba (Platymiscium trinitatis Benth.) em substrato constituído por latossolo amarelo textura argilosa, coletado após a queima da vegetação.

\begin{tabular}{|c|c|c|c|c|}
\hline \multirow[b]{2}{*}{ Tratamentos } & \multicolumn{3}{|c|}{ Dias após a repicagem*1 } & \multirow[b]{2}{*}{126} \\
\hline & 31 & 64 & 101 & \\
\hline \multicolumn{5}{|c|}{ Comprimento do caule $(\mathrm{cm})$} \\
\hline Testemunha & 11,0 & 12,5 & 17,5 & 19,9 \\
\hline $\mathrm{N}$-mineral & 11,0 & 12,9 & 14,9 & 19,9 \\
\hline Estirpe 512 & 10,0 & 11,8 & 13,8 & 16,4 \\
\hline Estirpe 524 & 9,1 & 10,9 & 14,3 & 15,9 \\
\hline Estirpe 525 & 10,4 & 11,1 & 15,8 & 18,3 \\
\hline Estirpe 541 & 9,6 & 11,5 & 15,1 & 17,9 \\
\hline Estirpe 590 & 10,6 & 12,3 & 17,1 & 19,6 \\
\hline Teste de F & $0,73 \mathrm{~ns}$ & $0,69 \mathrm{~ns}$ & $1,65 \mathrm{~ns}$ & $1,28 \mathrm{~ns}$ \\
\hline Coef. Var. (\%) & 22,84 & 21,16 & 19,99 & 21,26 \\
\hline \multicolumn{5}{|c|}{ Diâmetro do colo $(\mathrm{mm})$} \\
\hline Testemunha & $1,7 a b$ & 2,6 & 3,3 & $3,5 a b$ \\
\hline $\mathrm{N}$-mineral & $1,6 \mathrm{~b}$ & 2,5 & 2,8 & 3,7 a \\
\hline Estirpe 512 & $2,1 \mathrm{a}$ & 2,4 & 2,7 & $3,0 a b$ \\
\hline Estirpe 524 & $1,6 a b$ & 2,3 & 2,8 & $2,9 a b$ \\
\hline Estirpe 525 & $1,5 \mathrm{~b}$ & 2,2 & 2,7 & $2,7 \mathrm{~b}$ \\
\hline Estirpe 541 & $1,8 a b$ & 2,5 & 2,9 & $3,1 \mathrm{ab}$ \\
\hline Estirpe 590 & $1,9 a b$ & 2,4 & 3,2 & $3,3 a b$ \\
\hline Teste de F & $3,15^{*}$ & $0,76 \mathrm{~ns}$ & $2,20 \mathrm{~ns}$ & $2,30^{*}$ \\
\hline Coef. Var. $(\%)$ & 16,13 & 19,30 & 16,41 & 21,01 \\
\hline
\end{tabular}

$" 1$ - Médias seguidas de mesma letra na mesma coluna, não diferem entre si pelo teste de Tukey ao nível de $5 \%$ de probabilidade. $n s=$ não significativo . 
Tabela 3. Efeito da inoculação com rizóbios e da adubação nitrogenada na biomassa $(\mathrm{g})$ de mudas de macacaúba (Platymiscium trinitatis Benth.) repicadas para solo latossolo amarelo textura argilosa, coletado após a queima da vegetação.

\begin{tabular}{lccccc}
\hline & \multicolumn{2}{c}{\begin{tabular}{c} 
Peso da parte aérea \\
\cline { 2 - 3 } Tratamentos
\end{tabular}} & $\begin{array}{c}\text { Peso das } \\
\text { raizes secas }\end{array}$ & $\begin{array}{c}\text { Matéria seca } \\
\text { total }\end{array}$ & $\begin{array}{c}\text { Relação raiz/ } \\
\text { parte aérea }\end{array}$ \\
\cline { 2 - 3 } Testemunha & 4,73 & 1,59 & 0,63 & 2,22 & 0,40 \\
N-mineral & 4,30 & 1,40 & 0,50 & 1,89 & 0,38 \\
Estirpe 512 & 3,04 & 1,03 & 0,40 & 1,42 & 0,41 \\
Estirpe 524 & 3,86 & 1,27 & 0,49 & 1,77 & 0,40 \\
Estirpe 525 & 3,20 & 1,05 & 0,37 & 1,42 & 0,36 \\
Estirpe 541 & 3,56 & 1,22 & 0,54 & 1,77 & 0,44 \\
Estirpe 590 & 3,89 & 1,24 & 0,37 & 1,61 & 0,33 \\
Teste de F & $0,86 \mathrm{~ns}$ & $0,84 \mathrm{~ns}$ & $1,61 \mathrm{~ns}$ & $1,02 \mathrm{~ns}$ & $0,86 \mathrm{~ns}$ \\
Coef. Var. (\%) & 44,61 & 44,59 & 43,17 & 42,45 & 27,48 \\
\hline
\end{tabular}

" - Médias seguidas de mesma letra na mesma coluna, não diferem entre si pelo teste de Tukey ao nível de $5 \%$ de probabilidade. $n s=$ não significativo.

tratamento que recebeu a inoculação com a estirpe INPA 524. O peso específico dos nódulos foi baixo, variando entre 0,10 e $1,67 \mathrm{mg} /$ nódulo.

Souza (1992) estudou os efeitos de niveis crescentes de matéria orgânica no substrato de produção de mudas de três leguminosas florestais inoculadas, utilizando composto de lixo urbano como fonte orgânica e constatou que o incremento nos níveis de matéria orgânica no substrato resultou em drástica redução no número e peso dos nódulos desenvolvidos nas mudas, o que foi relacionado a uma maior disponibilidade de $\mathrm{N}$ mineralizado para as plantas. Tais resultados evidenciam que nas condições deste

Tabela 4. Efeito da inoculação com rizóbios e da adubação nitrogenada na nodulação de mudas de macacaúba da terra firme (Platymiscium trinitatis Benth.) repicadas para substrato constituído por latossolo amarelo coletado após a queima da vegetação.

\begin{tabular}{lcccc}
\hline Tratamentos & $\begin{array}{c}\text { Freqüência de } \\
\text { mudas noduladas } \\
(\%)\end{array}$ & $\begin{array}{c}\text { Peso dos } \\
\text { nódulos secos } \\
(\mathrm{mg})\end{array}$ & $\begin{array}{c}\text { Número de } \\
\text { nódulos/planta }\end{array}$ & $\begin{array}{c}\text { Peso específico dos } \\
\text { nódulos (mg/nódulo) }\end{array}$ \\
\hline Testemunha & 14,3 & 3 & 4 & 0,75 \\
N-mineral & 28,6 & 3 & 2 & 1,67 \\
Estirpe 512 & 0 & 0 & 0 & 0 \\
Estirpe 524 & 42,9 & 4 & 4 & 0,10 \\
Estirpe 525 & 14,3 & 3 & 6 & 0,50 \\
Estirpe 541 & 14,3 & 23 & 15 & 1,53 \\
Estirpe 590 & 0 & 0 & 0 & 0 \\
\hline
\end{tabular}


experimento, possivelmente, o nitrogênio não foi um elemento limitante ao crescimento das mudas, o que pode ser relacionado com os niveis elevados de fertilidade do substrato e com a taxa de matéria orgânica do solo, superior a $6 \%$. Sabe-se que $98 \%$ do $\mathrm{N}$ do solo disponivel para as plantas encontrase na matéria orgânica do solo (Sanchez, 1976), e a presença de $\mathrm{N}$ disponível, mineralizado da matéria orgânica por reações de amonificação e nitrificação (Barruenco et al., 1985), tem forte efeito inibidor para o estabelecimento e desenvolvimento de nódulos em leguminosas (Hallsworth, 1958; Robson, 1978). Novos estudos à serem efetuados em substratos com menor fertilidade e menores níveis de matéria orgânica poderão gerar informações adicionais sobre o potencial fixador de nitrogênio desta importante espécie madeirável da Amazônia.

\section{CONCLUSÕES}

Neste trabalho demonstrou-se que a germinação de sementes não é um fator silvicultural que limite o aproveitamento da "macacaúba" em regime de plantio intensivo. As sementes apresentaram elevada viabilidade, atingindo $86,0 \%$ de germinação, iniciada aos 4 dias após a semeadura e estendendo-se por 37 dias, revelando um pico de germinação aos 12 dias e IVE de $21,90(n=200)$. Entretanto a espécie apresentou baixas taxas de crescimento na fase viveiro e não respondeu à inoculação das mudas com rizóbios em latossolo amarelo coletado após a queima da vegetação. $\mathrm{O}$ incremento de comprimento do caule e diâmetro do colo das plantas foi de $2,49 \mathrm{~cm} / \mathrm{mês}$ e $0,45 \mathrm{~mm} / \mathrm{mês}$ sob condições de enviveiramento.

\section{Bibliografia citada}

Allen, O.N.; Allen, E.K. 1981. The leguminosae, a source book of characteristics, uses and nodulation. Univ. Wiscon$\sin$ Press, Madison, $812 \mathrm{p}$.

ATIK 1992. Seeds and plant propagation. Agroforestry Technology Information Kit №, 5, International Institute of Rural Reconstructions/Departament of Environment and Natural Resources/Ford Fundation. Manila, Philippines, 106p.

Benincasa, M.M.P. 1988. Análises de crescimento de plantas (Noções básicas). FUNEP, Jaboticabal. 42p.

Barbosa, O. 1968. Alguns aspectos de sementeiras e viveiros florestais. Rev, $d a$ Madeira, p.13-19.

Barruenco, C.R.; Garcia, F.S.; Subramanian, P. 1985. La fijación de nitrogeno atmosférico: una biotecnologia en la produccion agraria. Temas monográficos, Centro de Edafologia y Biologia Aplicada, Salamanca, Espanha, p.11-71.

Corrêa, M.P. 1926. Dicionário das plantas úteis do Brasil e das exóticas cultivadas. Ministério da Agricultura/ IBDF, 1984, Brasilia, Vol. 5, 687p.

Dobereiner, J. 1967. Efeito da inoculação de sementeiras de sabiá (Mimosa caesalpiniaefolia) no estabelecimento e desenvolvimento de mudas no campo. Pesquisa Agropecuária Brasileira, 2:301305.

EMBRAPA 1979. Manual de métodos de análise de solo. Serviço Nacional de Levantamento e Conservação de Solos. EMBRAPA-SNLCS, Rio de Janeiro, 98p. 
Franco, A.A.; Silva, G.G. 1985. Potential of the Rhizobium symbiosis in tree legumes. In: Freire, J.R.J.; Falcão, C.P.B. (Eds.). Proceedings of the workshop on Rhizobium/ legume inoculants. MIRCEN, Porto Alegre-RS, p.111-129.

Franco, A.A.; Faria, S.M. 1997. The contribution of $\mathrm{N}_{2}$-fixing tree legumes to land reclamation and sustainability in the tropics. Soil Biol. Biochem., 29(5/6):897-903.

Hallsworth, E.G. 1958. Nutritional factors affecting nodulation. In: Hallsworth, E.G. (Ed.). Nutrition of the legumes. Butterworths Scient. Publ,, London. p.183-201

Moreira, F.M.S.; Silva, M.F,; Faria, S.M. 1992. Occurrence of nodulation in legume species in the Amazon region of Brazil. New Phytol., 121:563-570.

Moreira, F.M.S.; Moreira, F.W. 1996. Caracteristicas da germinação de sementes de 64 espécies de leguminosas florestais nativas da Amazônia, em condições de viveiro. Acta Amazonica, 26(1/2):3-16.

Pásztor, Y.P.C. 1963. Métodos usados na colheita de sementes. Silvicultura em São Paulo. Boletim Técnico do Serviço Florestal, 1(2):305-323.

Pelczar, M.; Reid, R.; Chan, E.C.S. 1980. Caracterização e classificação de microrganismos. In: Pelczar, M.; Reid, R.; Chan, E.C.S. (Eds.) Microbiologia. MacGraw-Hill do Brasil, São Paulo, Vol. 1: p.43-56.

Popinigis, F. 1977. Fisiologia da semente. Ministério Agricultura, AGIPLAN, Brasília. 289p.
Ribeiro Júnior, W.Q. 1985. Eficiência e competitividade de estirpes de Rhizobium sp. para Albizia lebbek (L.) Benth e Enterolobium contortisiliquum (Velozo) Morong, em latossolo ácido. Dissertação de Mestrado. Universidade Federal Rural do Rio de Janeiro, Seropédica, Rio de Janeiro. 160p.

Robson, A.D. 1978. Mineral nutrients limiting nitrogen fixation in legumes. In: Andrew, C.S.; Kamprath, E.J. (Eds) Mineral nutrition of legumes in tropical and subtropical soils. CSIRO, Melbourne. p.277-293.

Sanchez, P.A. 1976. Nitrogen. In: Wiley, J.; Sons (Eds.). Properties and management of soils in the tropics. New York. p. 184221.

Souza, L.A.G. 1992. Seleçào em solos ácidos de Bradyrhizobium para leguminosas arbóreas e efeito do composto usado no substrato para produção de mudas noduladas. Dissertação de Mestrado, Universidade Federal do Rio Grande do Sul, Porto Alegre, Rio Grande do Sul, 99p.

Souza, L.A.G.; Silva, M.F.; Moreira, F.W. 1994. Capacidade de nodulação de 100 leguminosas da região Amazônica. Acta Amazonica, 24(1-2):9-18.

Souza, L.A.G. 1996. A coleção de rizóbios do Laboratório de Microbiologia do Solo do INPA/CPCA. Boletim da ALAR, 13(1):4-8.

SUDAM/CTM 1979. Pesquisas e informações sobre espécies florestais da Amazônia. SUDAM, Belém. $111 \mathrm{p}$. 